\title{
Expansión a largo plazo de la capacidad de Generación: caso del mercado acoplado Perú-Ecuador
}

\author{
Fabiola Jara, Jaime E. Luyo, José Meza \\ Programa de Doctorado en Ciencias con Mención en Energética, Universidad Nacional de Ingeniería, Av. \\ Tupac Amaru 210, Rímac, Lima, Perú
}

Recibido el 18 de noviembre 2020. Revisado el 16 de diciembre 2020. Aceptado el 20 de diciembre 2020

DOI: https://doi.org/10.33017/RevECIPeru2020.0013/

\section{Resumen}

La interconexión de mercados eléctricos posibilita la optimización del uso de recursos energéticos y mejora la confiabilidad de los sistemas. Sin embargo, también genera una redistribución de los beneficios entre los agentes, lo cual conlleva nuevos problemas para la coordinación y regulación, por lo cual existe la necesidad de diseñar nuevas políticas de regulación basadas en análisis de los impactos de dicha interconexión. El objetivo del presente artículo es mejorar la comprensión de los efectos que tendrá la interconexión en parámetros como: la evolución del parque de generación electricidad en cada sistema eléctrico, la incorporación de centrales hidroeléctricas de gran tamaño especialmente en países que cuenten con complementariedad hídrica y la incorporación de centrales eléctricas a base de recursos energéticos renovables no convencionales. Para ello, se ha estructurado un modelo que hace uso de la teoría de dinámica de sistemas y se analizó el caso de la interconexión Perú - Ecuador, se concluye que las políticas de estado previamente aplicadas en ambos países han originado que los costos marginales de sus sistemas sean artificialmente bajos, con lo cual no se dan las señales necesarias para que ingrese nueva capacidad de generación en el corto plazo.

Descriptores: Interconexión, mercado eléctrico, política regulatoria, simulación, complementariedad hídrica

\section{Abstract}

In the last years at international level the interconnection of electrical markets is being carried out, which makes it possible to optimize the use of energy resources and improve the reliability of the systems. This process of electrical integration generates a redistribution of benefits among agents, which also generates new problems for coordination and regulation, so there is a need to design new regulation policies based on analysis of the impacts of market interconnection. cross-border The objective of this article is to improve the understanding of the effects that interconnection will have on parameters such as: the evolution of the electricity generation park in each electrical system, the incorporation of large hydroelectric power plants especially in countries that have water complementarity and incorporation of power plants based on non-conventional renewable energy resources. To do this, a model has been structured that makes use of the theory of systems dynamics and the case of the Peru-Ecuador interconnection was analysed, finding that the state policies previously applied in both countries have caused that the marginal costs of their systems they are relatively low, so that new generation would not enter in the short term; It was also concluded that although expanding hydroelectric capacity may seem the best solution given the water complementarity between the two countries, low marginal costs mean that the first plants to be installed are based on natural gas.

Keywords: Interconnection, electricity market, regulation policies, simulation, water complementarity

\section{Introducción}

En América Latina como en diferentes partes del mundo, muchos países han decidido interconectar sus sistemas de electricidad con los objetivos de:
Ganar eficiencia en el uso de sus recursos, mejorar la seguridad de suministro, reducirlos costos, entre otros. El logro de estos objetivos depende de una expansión adecuada de la capacidad de generación y transmisión, ya que se busca aprovechar de forma 
óptima los recursos energéticos aliviando así los picos de demanda. El comercio de electricidad se da a través de un esquema de acoplamiento de mercado, para que dos regiones obtengan beneficios de costo y capacidad de manera óptima, lo cual está sujeto a límites en la capacidad de interconexión, dichos esquemas se han aplicado en sistemas liberalizados a través de modificaciones en el diseño del mercado y en el marco regulatorio [1].

Sin embargo, no hay acuerdo sobre cuáles son las políticas correctas para mejorar la fiabilidad, ya que poca capacidad de generación pondría en peligro la seguridad del suministro y podría aumentar el poder del mercado de las empresas de generación; pero, por otro lado, demasiada capacidad costaría más de lo que la gente está dispuesta a pagar y esto afecta la capacidad de recuperar los costos de inversión y se desincentiva nuevas inversiones en generación y transmisión. Asimismo, el proceso de liberalización de los mercados de electricidad crea desafíos para la planificación de la expansión, ya que se requiere un alto nivel de coordinación entre los agentes para desarrollar un sistema en el que las capacidades de generación y transmisión sean conjuntamente óptimas. Además, las inversiones en generación y transmisión no solo son interdependientes, sino que también se ven afectadas por el marco regulatorio. Por lo tanto, se deben enviar señales económicas para coordinar ambas decisiones de inversión [2].

En la actualidad muchos países con planes de interconexión intentan resolver los problemas de la remuneración de la capacidad de manera unilateral, y estos difieren de un país a otro considerablemente, ya que toman en cuenta factores de acuerdo con su realidad nacional, no teniendo en cuenta un análisis conjunto de los mercados que se planea interconectar, lo cual afecta a la interconexión ya que los mecanismos de capacidad no coordinados pueden distorsionar el comercio transfronterizo [3]. En este caso, los flujos y precios de la electricidad pueden verse afectados negativamente por estas diferencias, por esta razón se debe implementar políticas coordinadas, para que no se presente el caso de que un mercado subsidie al otro. Por lo tanto, los mecanismos de capacidad de mercado deben abrirse a la participación transfronteriza explícita para minimizar las distorsiones en el comercio transfronterizo, así garantizar la reducción de los costos de suministro a largo plazo [4].

El objetivo de la interconexión transfronteriza de electricidad es principalmente la optimización en el uso de recursos lo cual conlleva una reducción de costos de generación; sin embargo, los mecanismos de capacidad generan inquietudes acerca de la confiabilidad, debido a que las rentas de congestión de la interconexión se dividen entre ambos países, y esto puede originar que las inversiones sean difíciles asumir para un generador exportador en el país exportador ya que estaría asumiendo una obligación que esta fuera de su control en el país importador [5]

Estos eventos afectan la eficiencia de la interconexión a corto y a largo plazo por eso es importante estudiarlos mediante diferentes escenarios, teniendo en cuenta: i) los mecanismos de capacidad, ii) la participación en el mercado interconectado y iii) características propias del mercado nacional.

El objetivo de la presente investigación es mejorar la comprensión de los efectos que tendrá la interconexión de mercados eléctricos transfronterizos en los siguientes puntos:

- La evolución del parque de generación electricidad en cada sistema eléctrico.

- La incorporación de centrales hidroeléctricas de gran tamaño; en países que cuenten con complementariedad hídrica.

- La incorporación de centrales eléctricas a base de recursos energéticos renovables no convencionales.

Para alcanzar dicho objetivo se ha desarrollado el modelo propuesto por Jara et al [6], el cual hace uso de la teoría de dinámica de sistemas para interrelacionar:

i. Un modelo de expansión de la generación con un horizonte de simulación de largo plazo el cual sea suficiente para observar la evolución de las diferentes tecnologías de generación y cuenta con una resolución mensual que permita observar las variaciones estacionales de la oferta debidas a la hidrología.

ii. Un modelo de despacho económico de corto plazo para determinar el impacto en los costos marginales que afectarán la rentabilidad de los nuevos proyectos de generación, así como de las líneas de interconexión.

El análisis realizado se centra en el caso particular de Perú y Ecuador, en la sección 2 se hace una descripción del modelo empleado, en la sección 3 se presentan los resultados del análisis realizado para el caso de la interconexión entre Perú y 
Ecuador; Finalmente, en sección 4 se presentan las conclusiones.

\section{Metodología}

Para analizar el efecto de la interconexión de dos sistemas eléctricos se desarrolló un modelo que consideró el desarrollo dinámico del parque de generación y transmisión, Ver Figura 1.

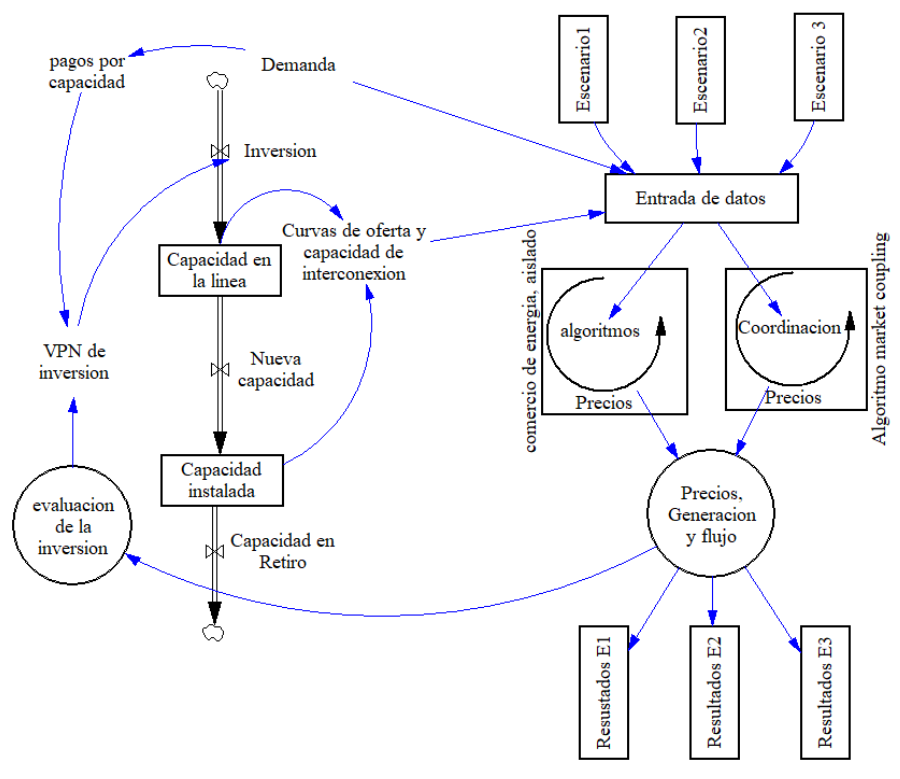

Figura 1. Metodología Propuesta

El modelo propuesto consta de dos submodelos: un modelo para el despacho de corto plazo y evaluación de inversiones y un modelo de expansión del sistema de largo plazo, los cuales interactúan y se retroalimentan de manera permanente.

La simulación comienza con el modelo de despacho, que proporciona la cantidad y costos marginales de la energía suministrada por cada planta de generación para cubrir la demanda en cada uno de los países; así como, si los países están interconectados, la energía importada/exportada y las rentas de congestión. A continuación, dicha información es ingresada al modelo de expansión de capacidad y es el punto de partida para la simulación en dicho modelo, el cual sobre la base de indicadores de rentabilidad calcula la incorporación de nuevas plantas de generación por tipo de tecnología; así como, la expansión o no de la línea de interconexión. Finalmente, dicha información es retroalimentada al modelo de despacho para que inicialice nuevamente los cálculos para el siguiente periodo evaluado.

\subsection{Modelo de despacho y cálculo del VPN}

El modelo se basa en un algoritmo de despacho por orden de mérito de corto plazo que simula el despacho de energía por hora, tanto para su operación en forma aislada, con interconexión y con interconexión ilimitada, para ello toma en cuenta los principios de acoplamiento de mercado y desarrolla los siguientes procesos:

- Calcula la generación por fuente de cada país, en términos de energía eléctrica generada y costos marginales, flujos por las líneas de interconexión y rentas de congestión.

- Evalúa las inversiones, y calcula el valor presente neto (VPN) de las inversiones por cada tecnología.

La programación del despacho económico por tecnología de generación tiene la formulación básica:

$$
\begin{gathered}
\min \sum_{t}\left[\sum_{j} C V_{j}\left(G_{j}(t)\right)+C A(V(t+1))\right] \\
\sum_{j}\left(\begin{array}{c}
G_{j}(t)+G_{h}(t)=D(t) \pm F I(t)-G_{S}(t) \\
-G_{e}(t)-G_{S C}(t)
\end{array}\right)
\end{gathered}
$$

\section{Sujeto a:}

$|F I(t)| \leq F I m a x$

$V \min \leq V(t)<V \max$

$G_{j} \min \leq G_{j}(t)<G_{j} \min$

Donde:

$C V_{i} \quad$ : Costo variable de tecnologías de generación (no incluye hidro, eólico y solar)

CA : Costo de oportunidad del agua (generación hidro con embalse)

$\mathrm{V}(\mathrm{t}) \quad$ : Volumen total embalse al final del periodo $\mathrm{t}$

$\mathrm{FI}(\mathrm{t})$ : Flujo en el Interconector en el periodo $\mathrm{t}$

$G_{j}(t)$ : Generación termoeléctrica en el periodo $t$

$\mathrm{D}(\mathrm{t}) \quad$ : Demanda en el periodo $\mathrm{t}$

$G_{h}(t)$ : Generación hidroeléctrica en el periodo $\mathrm{t}$

$G_{S}(t):$ Generación solar fotovoltaica en el periodo $t$

$G_{e}(t)$ : Generación eólica en el periodo $t$

$G_{S C}(t)$ : Generación solar de concentración en el periodo $t$

La solución óptima a este problema es equivalente a ordenar los generadores de menor a mayor costo variable en una lista de mérito, el operador del sistema despacha las unidades en ese orden hasta cubrir la demanda en cada período. Este algoritmo fue implementado en MATLAB. 


\subsection{Modelo de Expansión}

El modelo de expansión simula la evolución del parque de generación, así como la disponibilidad que tendrán durante su operación. Para ello se han estructurado 3 módulos los cuales se listan a continuación:

a) Módulo de evaluación de la inversión

Se realiza la evaluación de la inversión de las tecnologías disponibles, para ello se tiene en cuenta el retardo por el proceso de obtención de permisos y la construcción hasta la puesta en operación de la central.

b) Módulo del ciclo de vida de la capacidad instalada

Se evalúa el ciclo de vida del parque de generación y la línea de interconexión, desde el momento de la inversión hasta el momento del retiro de la unidad, La capacidad instalada determina las curvas de oferta y la capacidad de la línea de interconexión, que son los datos que se exportan al modelo de despacho para calcular las cantidades de energía generada y costos marginales. Este módulo también evalúa un análisis de esquemas de pagos por capacidad que podrían ser implementados en los escenarios evaluados.

c) Módulo de regulación de reservorio

Este módulo simula la regulación de los reservorios disponibles en ambos países, y es un dato de entrada esencial para el algoritmo de despacho, pues provee la información de la capacidad de generación que se tienen en forma diaria.

Los módulos descritos según el planteamiento y están basadas en los modelos desarrollados en [7] al [9]. Para la implementación del modelo se utilizó el software VENSIM DSS, Versión 7.3.5.

\section{Resultados y Discusión de Resultados}

Los escenarios evaluados buscan estudiar el comportamiento de dos sistemas interconectados frente a las políticas de suficiencia en capacidad de generación y transmisión. Además, cabe mencionar que la presente investigación del comportamiento de los mercados eléctricos transfronterizos no busca hacer pronósticos, sino sirve para analizar los impactos de posibles escenarios futuros. A fin de evaluar las diferentes opciones, se han simulado 7 escenarios, los cuales se listan en la Tabla I.

Tabla I: Escenarios evaluados

\begin{tabular}{|c|c|c|c|c|}
\hline Escenario & $\begin{array}{l}\text { Interconexión } \\
\text { (MW) }\end{array}$ & $\begin{array}{l}\text { Pagos por } \\
\text { Capacidad }\end{array}$ & $\begin{array}{c}\text { Precio de petróleo en } \\
\text { Ecuador } \\
\text { (US\$/galón) } \\
\end{array}$ & $\begin{array}{c}\text { Precio del Gas Natural en } \\
\text { Perú } \\
\text { (US\$/MMBTU) }\end{array}$ \\
\hline E01 & \multirow{6}{*}{$\begin{array}{l}\text { 2023: } 500 \\
2033:+500\end{array}$} & \multirow{4}{*}{$\begin{array}{l}\mathrm{P}: \mathrm{Si} \\
\mathrm{E}: \mathrm{Si}\end{array}$} & \multirow{2}{*}{ 2018-2045: 0.79} & 2018: 1.25, 2021-2045: 2.77 \\
\hline E02 & & & & Sin subsidio \\
\hline E03 & & & \multirow{2}{*}{$\begin{array}{l}\text { 2018: } 0.79,2023: 1.42 \\
\text { 2030: } 1.52,2040: 1.59\end{array}$} & 2018: 1.25, 2021-2045: 2.77 \\
\hline E04 & & & & Sin subsidio \\
\hline E05 & & P: Si, E: No & \multirow{3}{*}{ 2018-2045: 0.79} & \multirow{3}{*}{$\begin{array}{l}\text { 2018: } 1.25 \\
\text { 2021-2045: } 2.77\end{array}$} \\
\hline E06 & & P: No, E: Si & & \\
\hline E07 & llimitada & $\mathrm{P}: \mathrm{Si}, \mathrm{E}: \mathrm{Si}$ & & \\
\hline
\end{tabular}

Nota: P: Perú, E: Ecuador

El escenario E01 es el escenario base de la interconexión. Se prevé que el precio del GN (gas natural) para generación en el Perú continúe definido por la política de declaración actuales con lo cual el precio de GN sería de 1.25 US\$/MMBTU para el periodo 2018 - 2020, y a partir del año 2021, se asume que, según el mecanismo propuesto en el informe sobre la problemática del régimen de declaración de precios de GN [10], el precio de GN se situé en el nivel del precio para generadores eléctricos, el cual asciende a 2.77 US\$/MMBTU [11] manteniéndose este precio subsidiado de GN para generación eléctrica hasta el final del periodo de análisis.
En el caso del Ecuador, se prevé que la política de subsidio a los derivados de petróleo continúe, con lo cual el precio petróleo será de 0.79 US\$/Galón y el costo de generación de electricidad con residual y Diesel sea de 42 US $\$ / M W h$ y 90 US $\$ / M W h$, respectivamente.

Asimismo, en

este escenario se prevé la interconexión entre los sistemas eléctricos en el año 2023 con una capacidad de $500 \mathrm{MW}$ y una posterior ampliación a 1000 MW hacia el año 2023; también, se prevé el mecanismo de pagos por capacidad se seguirá empleando en ambos países. A continuación, se describen los principales resultados del escenario 
E01 y los resultados para el resto de los escenarios se presentan en el apéndice $B$.

\subsection{Costos Marginales Resultantes}

En el sistema eléctrico peruano se observa que en el periodo 2018 - 2020, el CMg (costo marginal) resultante tiene una variación entre los 8 y 15 USD/MWh en época de avenida y estiaje, respectivamente, esto debido a la distorsión de precios causada por la sobre oferta de generación y la declaración libre del precio del GN.

En el periodo 2021 - 2026 a consecuencia de las modificaciones propuestas para la declaración del precio de GN para generación de electricidad el CMg se incrementaría, pasando a estar entre los 25 y 34 US $\$ / M W h$ en época de avenida y estiaje, respectivamente. El mayor efecto que tiene estos costos marginales artificialmente bajos es que hacen inviable la construcción de nuevas centrales cuyo efecto se ve reflejado en el aumento de $\mathrm{CMg}$ en época de estiaje para los siguientes periodos.

En el periodo 2027 - 2035, se observa que los costos marginales en época de avenida se mantienen en el rango de los 25 US $\$ / M W h$ mientras que en época de estiaje se elevan llegando a picos de entre 40 y 60 US $\$ / M W h$, esto debido a que la construcción de las nuevas centrales hidráulicas con capacidad de regulación tiene un periodo de obtención de permisos y construcción que están en el orden de los 8 años.

Finalmente, en el periodo 2036 - 2045 el CMg en época de estiaje estaría en el rango de los 46 US $\$ / M W h$ y 24 a 20 US $\$ / M W h$ para épocas de avenida, como se presenta en la Figura 2, dicha reducción es el resultado de la puesta en operación de proyectos de generación hidráulica, eólica y solar, cuya decisión de implementación es resultado del modelo de expansión, y permiten que por un lado se incremente la capacidad de generación con centrales hidráulicas con embalse y por otro lado, el aporte de las fuentes eólicas y solares hacen que se pueda disponer de mayor volumen de agua para regulación.

La Figura 3, presenta los costos marginales en Ecuador, en el periodo 2018 - 2023 existe una alta variabilidad en el $\mathrm{CMg}$ en épocas de avenida con 2 US $\$ / M W h$ y épocas de estiaje con 50 US $\$ / M W h$, esto se debe a que el parque de generación se compone principalmente de centrales hidroeléctricas de pasada (las cuales no permiten la regulación fuera de la época de avenida) y centrales térmicas que funcional con fuel oil o residual cuyos precios son subsidiados por el estado ecuatoriano. Para el periodo 2024 - 2033, se observa que esta variabilidad se va reduciendo paulatinamente como resultado de i) la incorporación de centrales térmicas con combustible subsidiado y ii) la interconexión con el sistema eléctrico peruano que permite la exportación de excedente en épocas de avenida y la importación de energía desde el Perú (basada en generación termina a GN subsidiada) en época de estiaje, con lo cual la variación en el CMg se reduce hasta los 35 y 50 US $\$ / M W h$ para épocas de avenida y estiaje, respectivamente.

Finalmente, para el periodo $2034-2045$, los costos marginales se estabilizan en el orden de los 38 US $\$ / M W h$ para época de avenida y 52 US $\$ / M W h$ para época de estiaje.

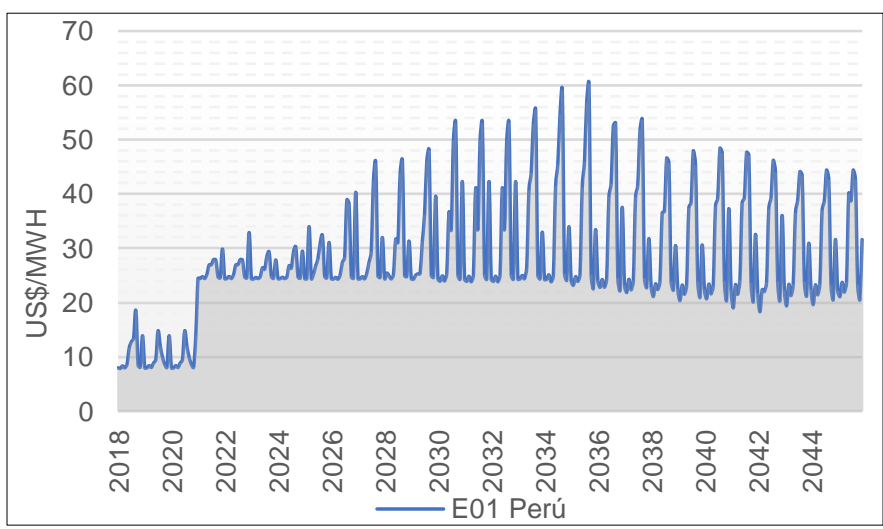

Figura 2: Costos Marginales en Perú para E01

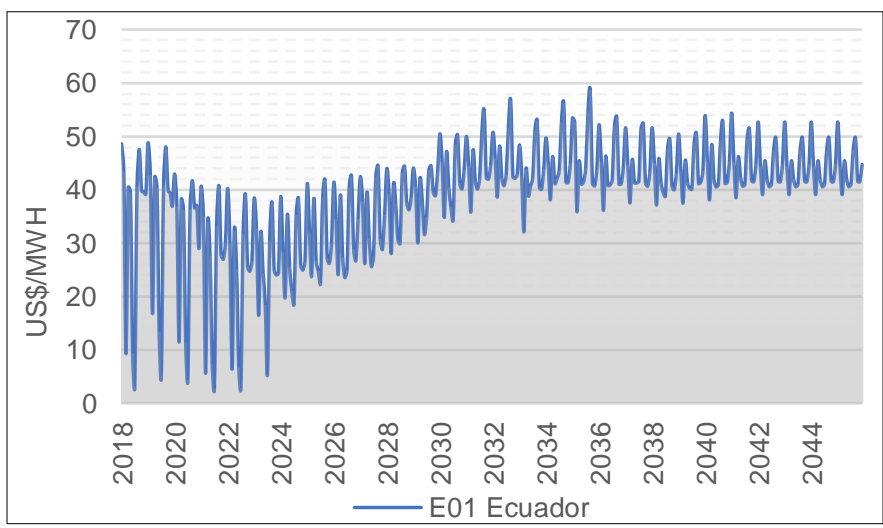

Figura 3: Costos Marginales en Ecuador para E01

\subsection{Flujo de Energía en la Interconexión}

La Figura 4 (a), presenta el intercambio de energía entre Perú y Ecuador para los dos primeros años del periodo 2023 - 2032, en general el sentido del intercambio de energía va desde el país con el menor $\mathrm{CMg}$ hacia el país con un mayor $\mathrm{CMg}$. El intercambio de Perú hacia Ecuador se produce los 
primeros meses del año cuando es época de avenida en Perú y por lo tanto su CMg es menor al de Ecuador, mientras que el intercambio de Ecuador hacia Perú se produce en la época de avenida en Ecuador durante los meses de julio y agosto, comprobándose con esto que existe una marcada complementariedad hidrológica entre ambos países. Sin embargo, el aspecto que más influiría en los intercambios es el bajo costo del GN para generación en el Perú, ya que provoca que los $\mathrm{CMg}$ en Perú en meses fuera del periodo de avenida (mayo, junio y de setiembre a diciembre) siga siendo menor que el CMg en Ecuador, por lo que el mayor volumen de intercambio se produce de Perú hacia Ecuador.

El efecto del bajo precio del GN en Perú se acentúa en el Periodo 2033 - 2045, como se presenta en la Figura 4 (b). Donde el flujo del intercambio de Perú hacia Ecuador se llega a presentar incluso durante algunas horas de la época de avenida en Ecuador.

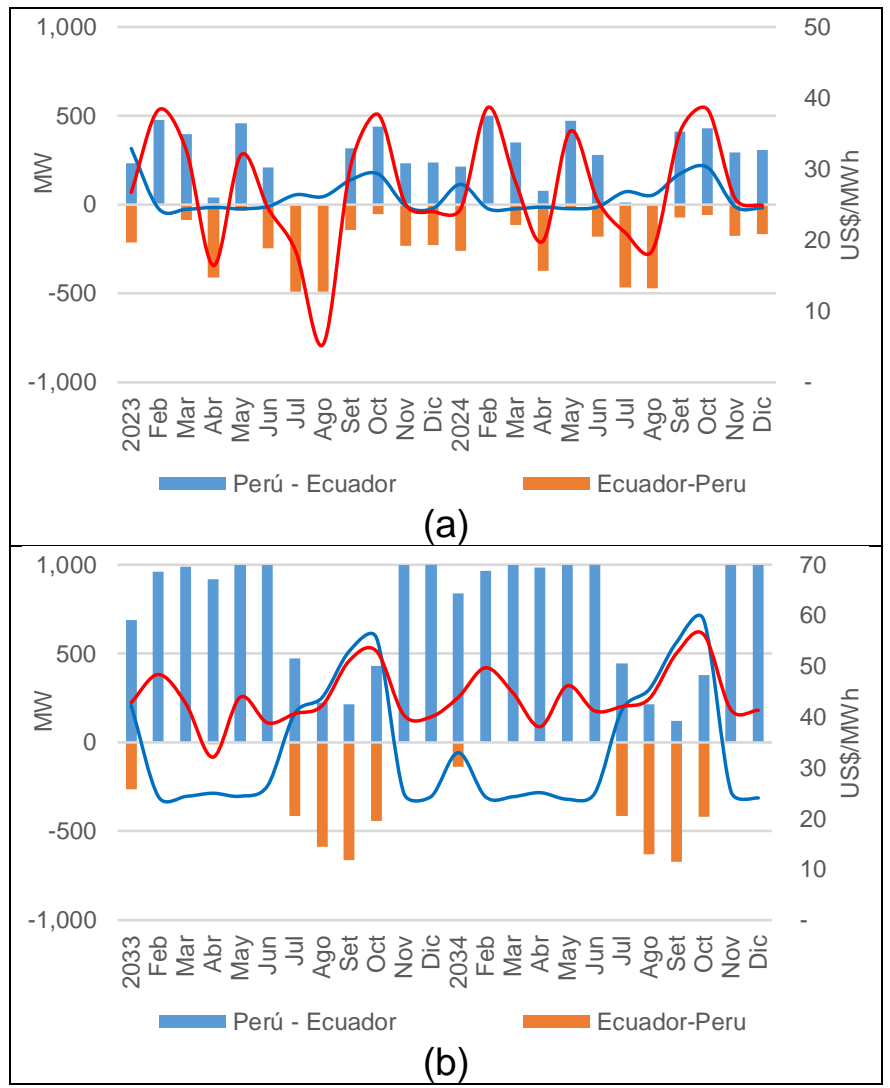

Figura 4: Flujo de energía en la interconexión en E01

\subsection{Capacidad Instalada de Generación}

La capacidad instalada de generación eficiente en el Perú (no se consideran plantas a Diesel ni de reserva fría) pasa de $10.1 \mathrm{GW}$ en 2018 a $27.9 \mathrm{GW}$ en 2045 , mientras que en Ecuador pasa de $7.2 \mathrm{GW}$ en 2018 a 17.3 GW en 2045 (para ecuador se considera la generación a base de petróleo residual o fuel oíl), Asimismo, se ha considerado el retiro de la única planta a carbón existente en Perú (140 MW) para el año 2023, ya que forma parte de la estrategia global de descarbonización de su propietario [12], Ver Figura 5

En términos de participación por tecnología, en el Perú se pasa de tener una componente de hidroelectricidad y GN de $48.7 \%$ y $43 \%$ en 2018 a $45.6 \%$ y $21.9 \%$ en 2045 , respectivamente. Mientras que las energías eólica, solar y biomasa pasan de representar un $3.7 \%, 2.8 \%$ y $0.4 \%$ en 2018 a $14 \%$, $17.2 \%$ y $1.2 \%$ en 2045 . Ver Figura 6.

Este cambio en la participación de las tecnologías es consecuencia directa del bajo costo del GN para generación en el Perú, pese a que se prevé un sinceramiento en la declaración del precio en el 2023, pasando de un costo variable de generación de 8 US $\$ / M W h$ a 24.4 US $\$ / M W h$, este precio sigue siendo bajo en relación con otras tecnologías.

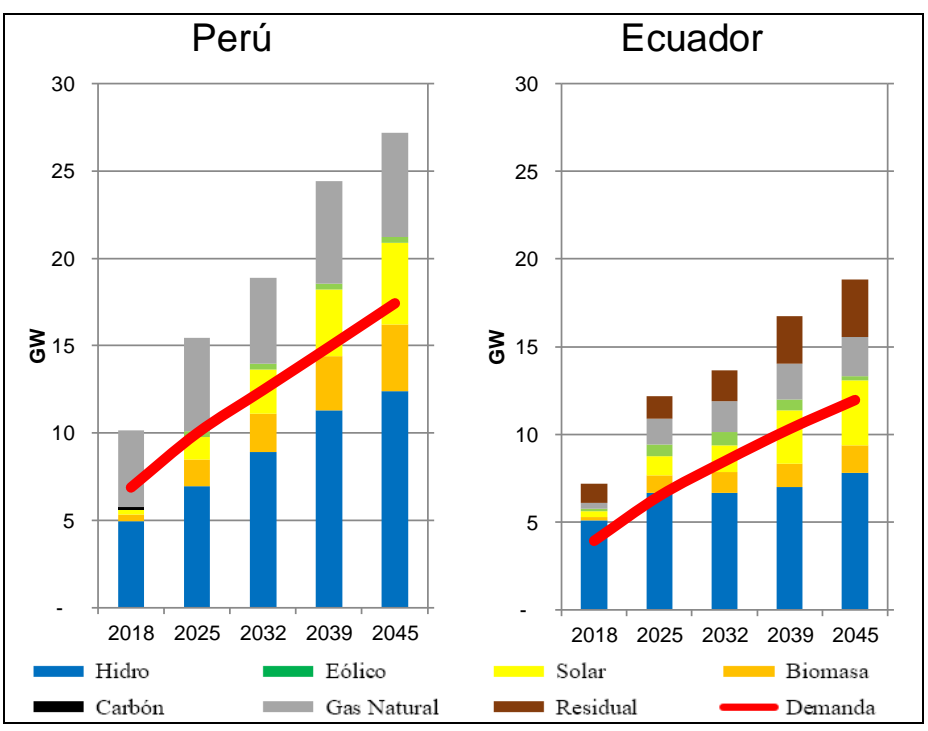

Figura 5: Evolución de la capacidad instalada de generación para E01

Por otra parte, en Ecuador se pasa de tener una componente de hidroelectricidad, residual y GN de $70.4 \%, 15.4 \%$ y $4.8 \%$ en 2018 a $41.5 \%, 17.4 \%$ y $11.8 \%$ en 2045 , respectivamente. Mientras que las energías eólica, solar y biomasa pasan de representar un $3 \%, 4.5 \%$ y $1.9 \%$ en 2018 a $8.3 \%$, $19.6 \%$ y $1.4 \%$ en 2045 , como se presenta en la Figura 7.

La variación en la participación de las tecnologías es consecuencia del bajo costo del combustible residual que es subsidiado por el estado ecuatoriano el cual mantiene un costo variable de generación de 42.4 US\$/MWh a lo largo del periodo de análisis; lo cual influye en un bajo $\mathrm{CMg}$ que, 
sumado a la posibilidad de importar electricidad a bajo precio, no incentiva las inversiones en plantas hidroeléctricas.

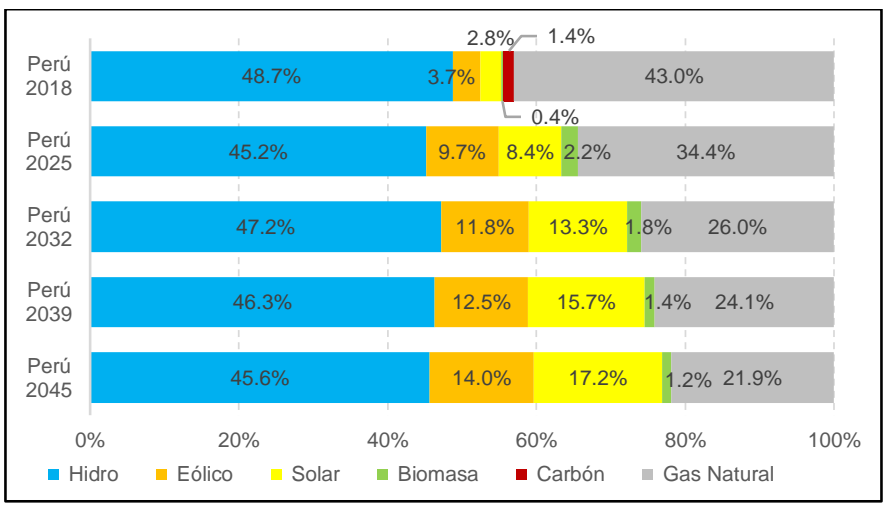

Figura 6: Evolución de la participación por tecnología en Perú para E01

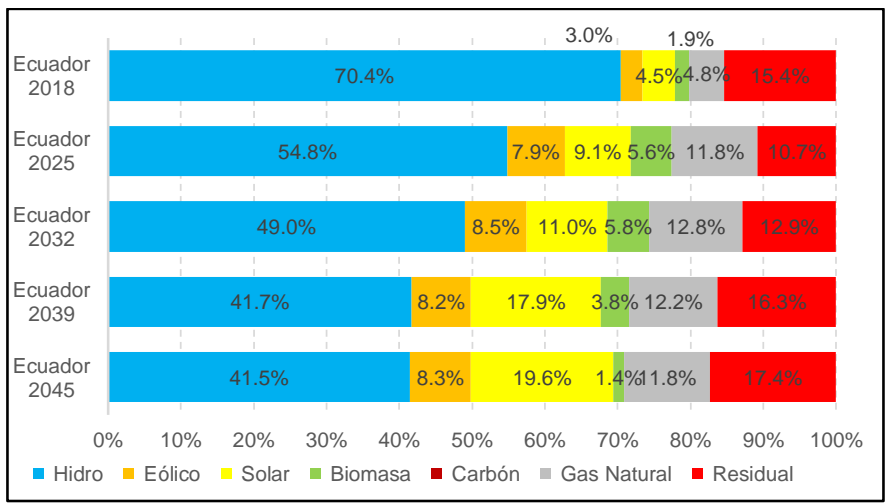

Figura 7: Evolución de la participación por tecnología en Ecuador para E01

\section{Conclusiones}

El modelo presentado en este articulo ayuda a comprender los nuevos desafíos para los reguladores del mercado eléctrico como consecuencia de la interconexión. Los resultados de la simulación muestran que la integración del mercado de electricidad puede traer beneficios en términos de menores costos de suministro y mejor uso de los recursos; sin embargo, estos beneficios dependen en gran medida del grado de interconexión y varían de un país a otro, dependiendo, entre otras cosas, del estado actual de sus parques de generación de electricidad y los márgenes de reserva con que cuenten.

En el caso de Perú y Ecuador, el efecto de la implementación unilateral de políticas de suficiencia de capacidad es que ambos sistemas tienen costos marginales artificialmente bajos que se sustentan en subsidios, en el caso del Perú al precio del GN y en el Ecuador al precio del combustible residual, además que ambos países implementaron políticas de inversión en centrales hidroeléctricas que no recuperarían su inversión con dichos costos marginales.

El efecto de la implementación de centrales hidroeléctricas y centrales renovables no convencionales es la reducción en el $\mathrm{CMg}$ del sistema, y el mejor aprovechamiento de recursos; sin embargo, como se mencionó anteriormente el $\mathrm{CMg}$ puede estar siendo influenciados por otras políticas energéticas y no dar la señal adecuada para la inversión, esto es más crítico en el caso de las centrales hidroeléctricas que requieren de un mayor monto de inversión y largo periodo de construcción, y sumado a esto que en los últimos años vienen presentándose reclamos socio ambientales en sus zonas de influencia.

Los subsidios en el precio de los combustibles conllevan a costos marginales artificialmente bajos, y originan que tecnologías que requieren altos costos de inversión no se realicen. Desde el punto de vista del usuario del país exportador el impacto negativo se duplica ya que por un lado al exportar electricidad el CMg se eleva y además está exportando un subsidio que sale de otra parte de la economía del país exportador.

Los mecanismos de pagos por capacidad incentivan las inversiones en tecnologías convencionales con potencia firme a ofrecer y de no implementar un mecanismo para su reconocimiento a centrales renovables no convencionales, podrían representar una barrera para la implementación de estas últimas.

El CMg promedio mensual en Ecuador tiene una gran correlación con su hidrología ya que en el periodo 2016 - 2019 han ingresado a su sistema una importante cantidad de centrales hidroeléctricas de pasada las cuales influenciaron a la baja el CMg de los próximos años. Asimismo, se observa que en los primeros cinco años de simulación no hay un efecto importante ya que la línea de interconexión recién se pone en funcionamiento el año 2023, lo cual tiene un efecto inmediato en la subida del CMg en Perú. Sin embargo, la característica predominante en ambos sistemas es el bajo costo de GN en el Perú y el combustible residual en Ecuador, que dependen de políticas energéticas que fueron adoptadas por sus respectivos gobiernos que en su momento buscaron la suficiencia de generación. Dichos precios son muy sensibles ya que en ambos países son los que determinan el $\mathrm{CMg}$ del sistema, con lo cual se estarían importando y exportando subsidios desde un país hacia el otro.

\section{Agradecimientos}


Los autores brindan un agradecimiento especial al

Fondo Nacional de Desarrollo Científico, Tecnológico y de Innovación Tecnológica (Convenio $n \circ 207-2015-F O N D E C Y T)$ por la beca de doctorado en ciencias con mención en energética.

\section{Apéndice A Tabla de Acrónimos}

\begin{tabular}{|l|l|}
\hline BTU & $\begin{array}{l}\text { British Thermal Unit / Unidad } \\
\text { Térmica Británica }\end{array}$ \\
\hline CMg & Costo Marginal \\
\hline
\end{tabular}

\begin{tabular}{|l|l|}
\hline GN & Gas Natural \\
\hline MM & Millones \\
\hline MW & Megavatio \\
\hline MWh & Megavatio-hora \\
\hline US\$ & Dólares Norteamericanos \\
\hline VPN & Valor Presente Neto \\
\hline
\end{tabular}

Apéndice B Resultados de Escenarios E02 - E07

120

80

40

กิ่

(a)
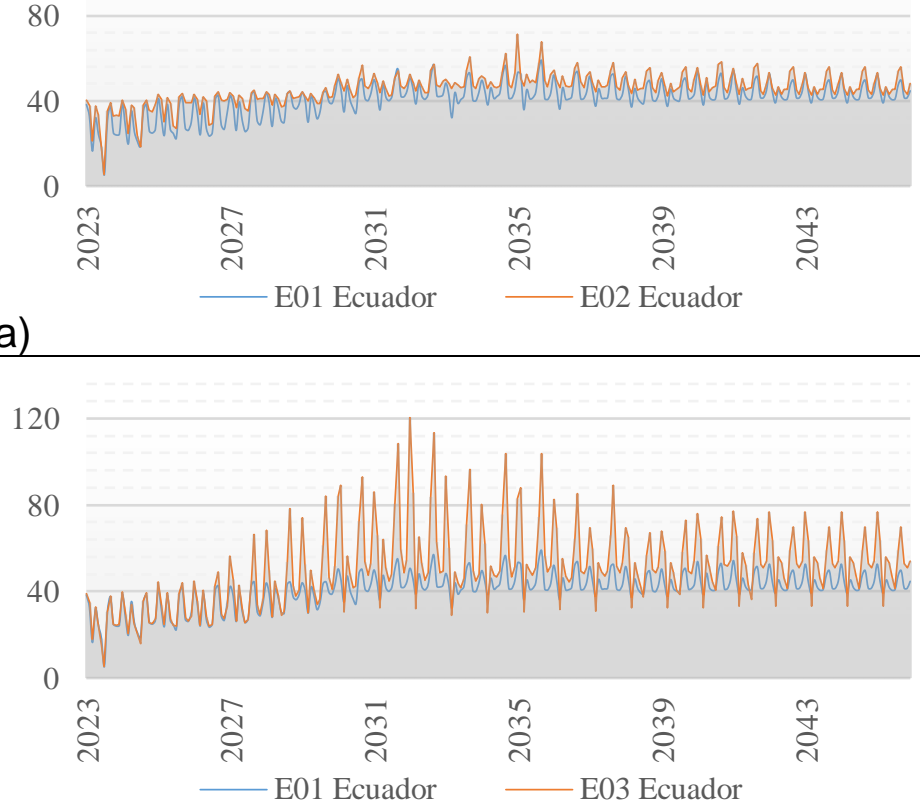

(b)

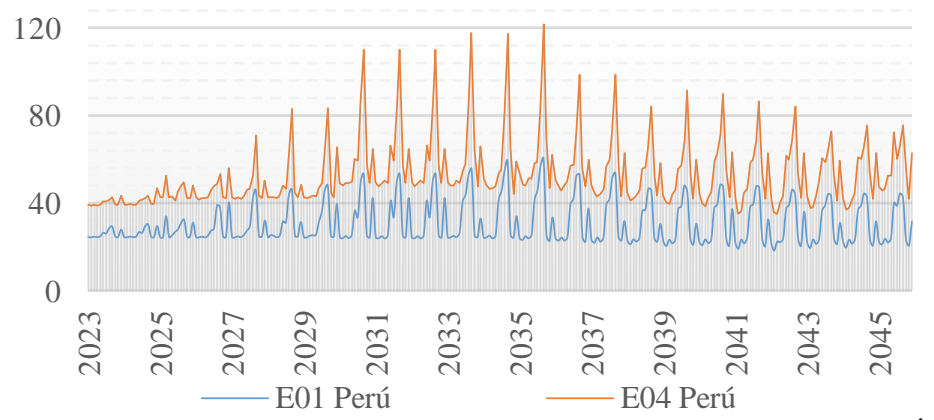

(c)

120

80

40

0

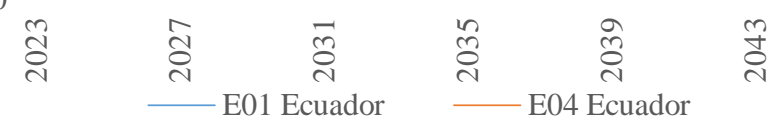

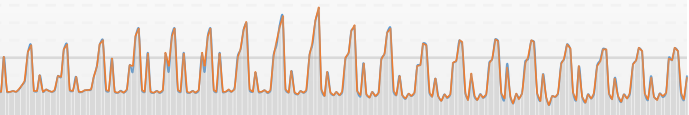

0

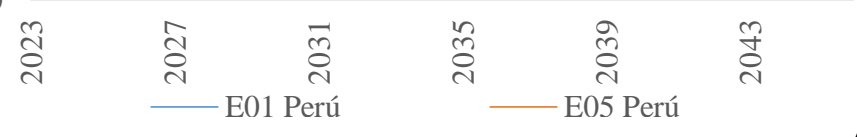

(d)

40

0

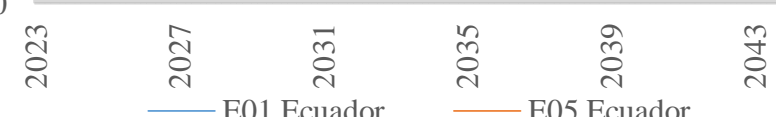


80

40

0

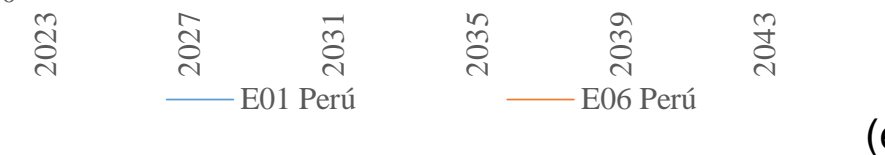

80

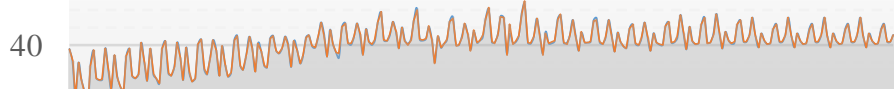

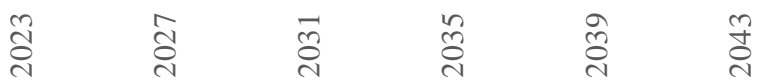

e)

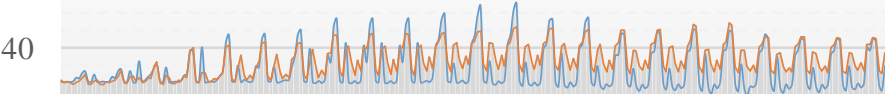

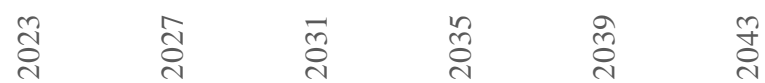
- E01 Perú E07 Perú
40 (1)

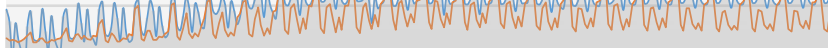
0

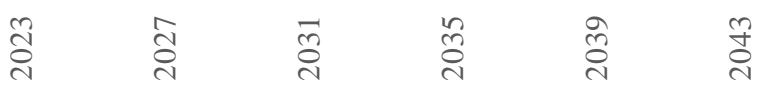
(f)

Figura A1: Variación en los costos marginales de los escenarios Evaluados (US\$/MWh)

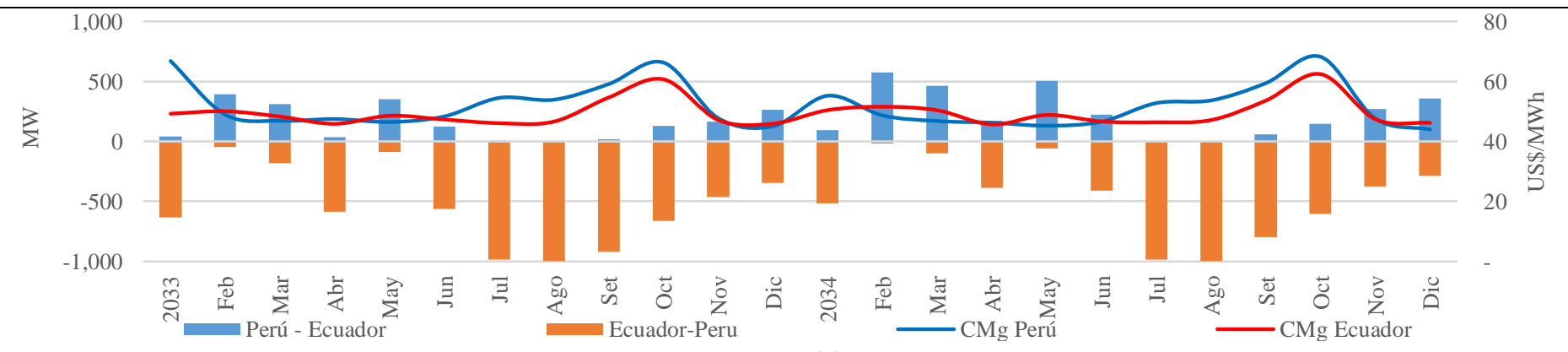

(a) E02

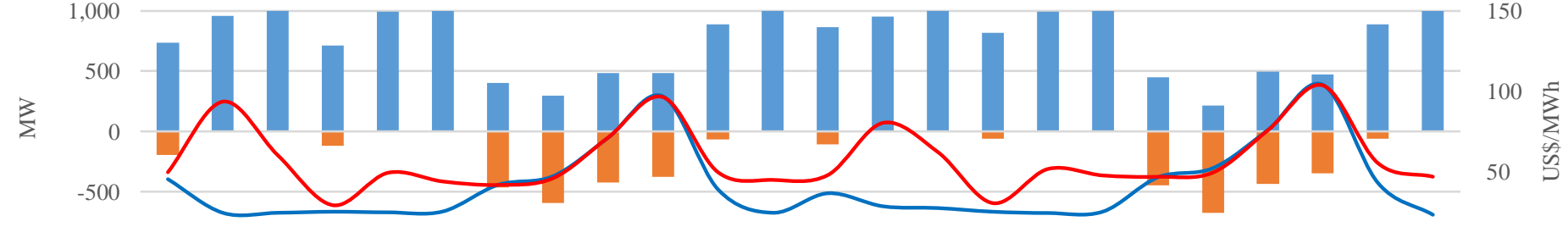

$-1,000$

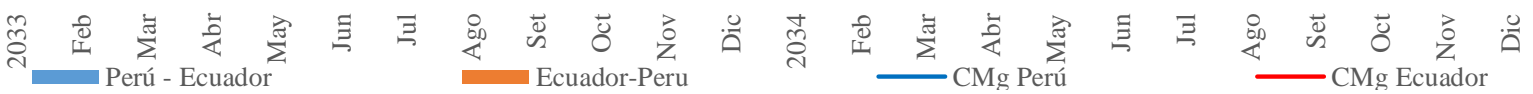

(b) E03

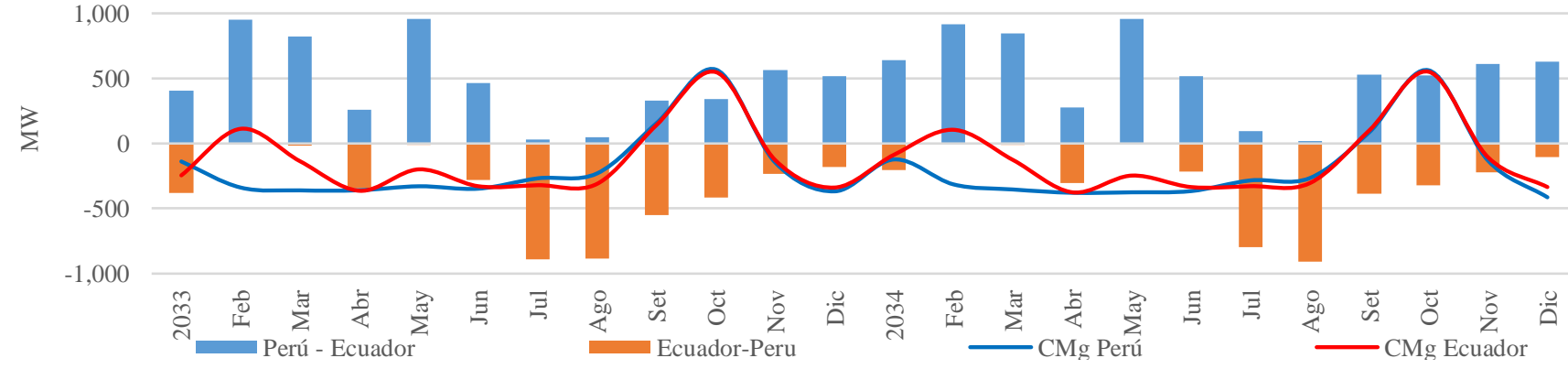

(c) E04 


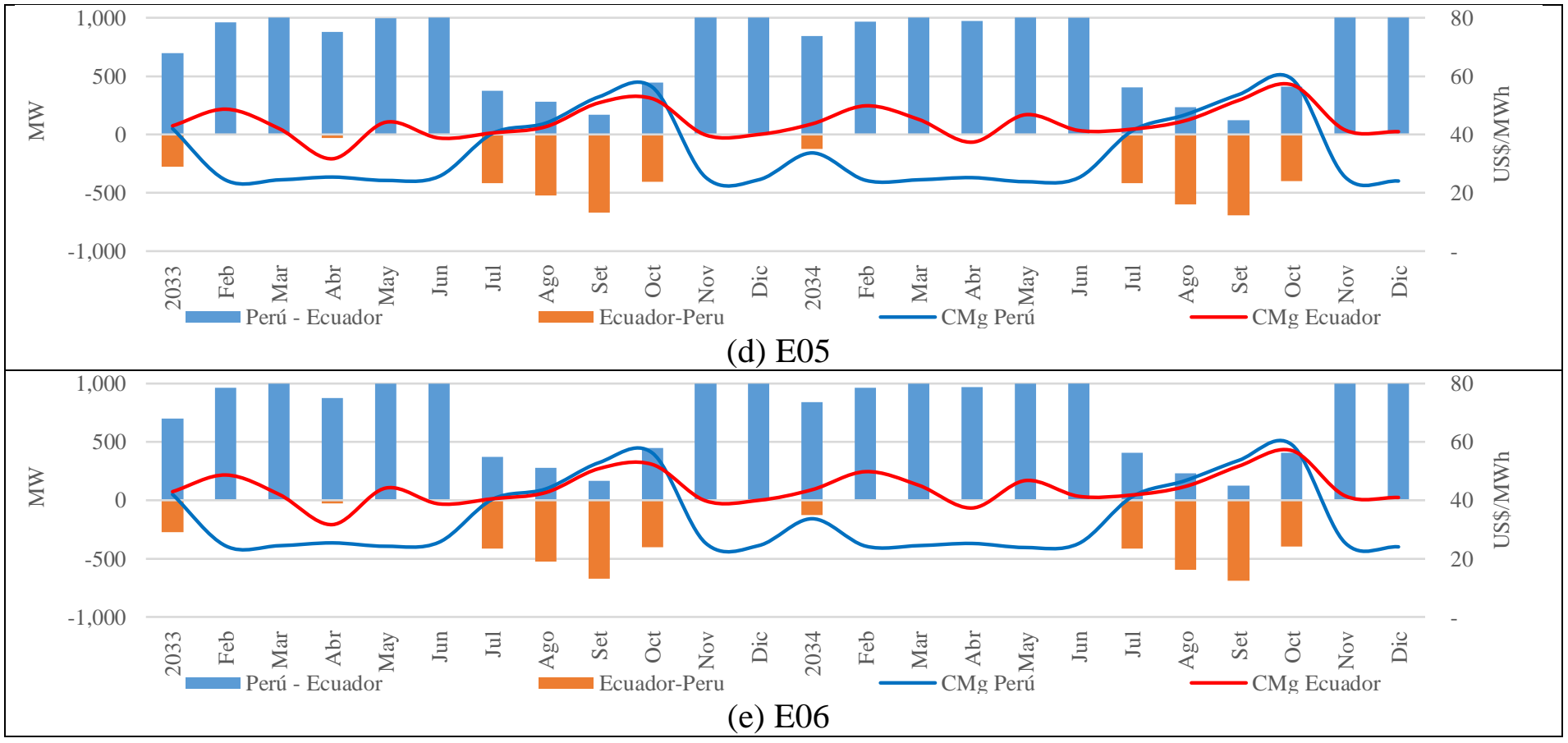

Figura A2: Flujo de energía en la interconexión en los escenarios Evaluados

\section{Referencias}

[1] T. Jamasb and M. Pollitt, "Security of supply and regulation of energy networks", Energy Policy, vol. 36, no. 12, pp. 45844589, dec. 2008.

[2] S. Stoft, "Problems of transmission investment in a deregulated power market" in Competitive electricity markets and sustainability, 2006, pp. 87-130.

[3] P. Cramton, A. Ockenfels and S. Stoft, "Capacity market fundamentals", Economics of Energy \& Environmental Policy, vol. 2, no. 2, pp. 27-46, sep 2013.

[4] P. Mastropietro, P. Rodilla and C. Batlle, "National capacity mechanisms in the european internal energy market: Opening the doors to neighbours", Energy policy, vol. 28, pp. 38-47, jul 2015.

[5] D. Finon, "Capacity Mechanisms and Cross-border Participation: the EU Wide Approach in Question. 2018", HAL, hal01687454, jun 2018.

[6] F. Jara et al., "Interconexión de Mercados Transfronterizos de Electricidad y la Necesidad de Políticas de Coordinación", Revista Tecnia, vol. 28, n 1, pp. 1-4, dic. 2018.

[7] C. Ochoa and O. Gore, "The Finnish power market: Are imports from Russia low-cost?", Energy Policy, vol. 80, pp. 122-132, jun. 2015.

[8] C. Ochoa and A. Van Ackere, "Does size matter? Simulating electricity market coupling between Colombia and Ecuador", Renewable and Sustainable Energy Reviews, vol. 50, pp. 1108-1124, jun. 2015.

[9] C. Ochoa and O. Gore, "Winners and losers of market coupling", Energy, vol. 80, pp. 522-534, jun. 2016.

[10] Ministerio de Energía y Minas, "Informe sobre Problemática del Régimen de Declaración de Precios de Gas Natural", Lima, Perú, 2019. [Online]. Available: http://www.minem.gob.pe/file/InformeGru podeGeneracionCRSE.pdf

[11] Online]. Available:

http://www.osinergmin.gob.pe/Resolucion es/pdf/2020/Informe-Tecnico-502-2020-

\section{GRT.pdf}

[12] ENGIE, "Memoria Anual 2019", Lima, Perú, 2019. Online]. Available: https://bit.ly/34iGZkp

E-mails: fabiolajaraca@gmail.com jeluyo@yahoo.es jose.meza.s@uni.pe 